\title{
ARLI4 as a Prognostic Biomarker in Non-Small Cell Lung Cancer
}

\author{
Binbin Zhang ${ }^{1, *}$ \\ Aiqun $\mathrm{Xu}^{2, *}$ \\ Dong $\mathrm{Wu}^{\mathrm{l}}{ }^{*}$ \\ Wanli $\mathrm{Xia}^{3, *}$ \\ Pulin $\mathrm{Li}^{\mathrm{l}}$ \\ Enze Wang' \\ Rui Han' \\ Peng Sun' \\ Sijing Zhou ${ }^{4,5}$ \\ Ran Wang (D)'
}

'Department of Respiratory and Critical Care Medicine, The First Affiliated Hospital of Anhui Medical University, Hefei, Anhui Province, People's Republic of China; ${ }^{2}$ Department of General Medicine, Hefei Second People's Hospital, Hefei, Anhui Province, People's Republic of China; ${ }^{3}$ Department of Thoracic Surgery, The First Affiliated Hospital of Anhui Medical University, Hefei, Anhui Province, People's Republic of China; ${ }^{4}$ Hefei Third Clinical College of Anhui Medical University, Hefei, Anhui

Province, People's Republic of China; ${ }^{5}$ Hefei Prevention and Treatment Center for Occupational Diseases, Hefei, Anhui Province, People's Republic of China

*These authors contributed equally to this work

Correspondence: Sijing Zhou Hefei Prevention and Treatment Center for Occupational Diseases, Hefei, 230022, People's Republic of China $\mathrm{Tel} / \mathrm{Fax}+86$ 55I-63672040

Email zhousijing@yeah.net

Ran Wang

Department of Respiratory and Critical

Care Medicine, The First Affiliated

Hospital of Anhui Medical University,

Hefei, 230022, People's Republic of China,

Tel/Fax +86-55I-6292-29I4

Email ranwangtjmu@hotmail.com
Purpose: Non-small cell lung cancer (NSCLC) is the leading cause of cancer-related deaths worldwide. The mechanisms underlying NSCLC initiation and progression require further investigation. The purpose of this study was to investigate the role of ADP ribosylation factor-like GTPase 14 (ARL14) related to the progression of NSCLC.

Patients and Methods: We analyzed the correlation between clinical characteristics and ARL14 expression using data from The Cancer Genome Atlas (TCGA). Kaplan-Meier analysis was conducted to evaluate the prognostic value of ARL14 in NSCLC. Functions of ARL14 were identified by enrichment analysis. The relationship between ARL14 expression and immune cell infiltration was also studied. Furthermore, ARL14 expression was examined using immunohistochemistry, and its clinical significance was analyzed in 120 patients with NSCLC.

Results: Our study revealed that the expression level of ARL14 in patients with NSCLC was higher than that in normal tissues. Using TCGA data, higher ARL14 expression in lung adenocarcinoma was associated with residual tumor $(\mathrm{P}=0.017)$, while it was associated with age $(\mathrm{P}=0.003)$ and $\mathrm{N}$ stage $(\mathrm{P}=0.009)$ in lung squamous cell carcinoma. Similar results were obtained from 120 patients with NSCLC. High ARL14 expression was associated with poor overall survival and progression-free survival in NSCLC. Multivariate analysis revealed that ARL14 was an independent risk factor for patients with NSCLC. Functional enrichment analysis indicated that ARL14 was related to the occurrence and development of tumors.

Conclusion: Increased ARL14 expression was considerably correlated with poor survival in NSCLC, and it might be a promising prognostic biomarker for NSCLC.

Keywords: ADP ribosylation factor-like GTPase 14, non-small cell lung cancer, prognosis, immunohistochemistry, bioinformatics

\section{Introduction}

Lung cancer is the most commonly diagnosed cancer worldwide and the leading cause of cancer-related deaths $(18.4 \%$ of the total cancer deaths $) .{ }^{1}$ Non-small cell lung cancer (NSCLC) accounts for $83 \%$ of all lung cancer cases with a 5-year survival rate of $23 \%$ after diagnosis. ${ }^{2}$ Lung adenocarcinomas (LUADs) and squamous cell cancers (LUSCs) are the most significant NSCLC subgroups. ${ }^{3}$ Despite the development of medical technology such as increasing immunotherapy, the prognosis for NSCLC remains poor, which highlights the need to identify oncogenic and tumor-suppressive genes related to patient prognosis as molecular biomarkers. $^{4-7}$

ADP ribosylation factor-like GTPase 14 (ARL14), also known as ARF7 and FLJ22595, is a protein-coding gene that participates in GTP binding and signal 


\section{Graphical Abstract}

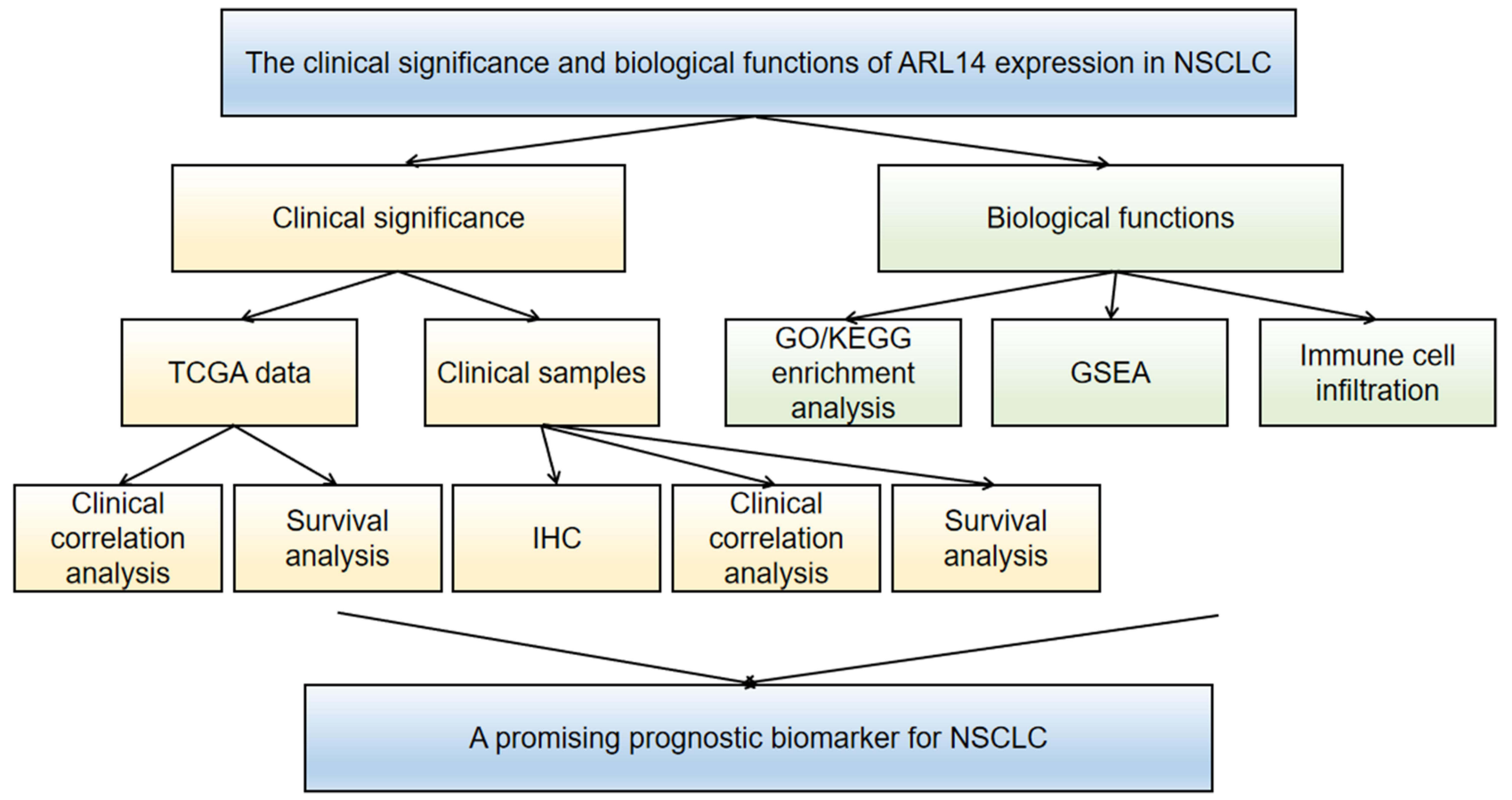

transduction. ${ }^{8,9}$ It is a member of the ADP ribosylation factor (ARF) family of GTP-binding proteins of the Ras superfamily. The primary function of ARF family proteins is to mediate the recruitment of cytosolic effectors to specific membrane compartments. ${ }^{10-12}$ As central control elements, GTPases participate in signal transduction pathways, which touch almost every aspect of cell biology. ${ }^{13}$ Recently, some studies have shown that ARL14 plays a key role in a wide range of biological processes such as intracellular protein transport and vesicle-mediated transport. ${ }^{14}$ The methylation level of ARL14 is positively related to poor survival in patients with bladder cancer. ${ }^{15}$ The homolog of ARL14, ARF3, is reported to be poorly expressed in gastric cancer and may act as a potential biomarker for gastric cancer prognosis. ${ }^{16}$ ARF6, a homolog of ARL14, can promote pancreatic cancer development by enhancing the Warburg effect. ${ }^{17}$ However, few studies on the relationship between ARL14 and cancer have been reported. Therefore, this study aimed to assess the level of ARL14 in NSCLC and investigate the correlation between ARL14 expression and prognosis and clinicopathological indicators of NSCLC.

We used LUAD and LUSC messenger RNA (mRNA) expression data from The Cancer Genome
Atlas (TCGA) database to compare the difference in ARL14 expression between tumor tissues and normal samples and evaluated the correlation between ARL14 expression levels and clinical pathological features. Immunohistochemistry (IHC) was also used to investigate the difference in ARL14 expression between tumor tissues and normal samples. We evaluated the prognostic value of ARL14 in NSCLC. We also investigated the biological pathways related to ARL14 using gene set enrichment analysis (GSEA). The correlation between ARL14 expression and immune infiltration revealed its possible functions. Our results suggest that ARL14 may act as a promising prognostic biomarker for NSCLC.

\section{Materials and Methods}

\section{Bioinformatics Analysis Based on TCGA Database}

mRNA expression data, corresponding clinical information, and survival data of TCGA-LUAD (594 samples, workflow type: HTSeq-FPKM) and TCGA-LUSC (551 samples, workflow type: HTSeq-FPKM) were obtained from TCGA database (https://cancergenome.nih.gov, 
free database, Table S1). The LUAD dataset included 535 tumor tissues and 59 normal tissues, whereas the LUSC dataset included 502 cancer tissues and 49 normal tissues (Table 1). A total of 517 patients with LUAD and 496 patients with LUSC had complete survival data.

\section{Sample Collection}

All patients were initially diagnosed with NSCLC. A total of 120 human NSCLC tissues and 120 adjacent normal lung tissues (samples were taken approximately more than $5 \mathrm{~cm}$ from the edge of the tumor) were fixed in formalin and embedded in paraffin for IHC after surgical removal.

Table I Correlation Between ARLI4 Expression and Clinicopathological Characteristics in LUAD and LUSC

\begin{tabular}{|c|c|c|c|c|c|c|}
\hline \multirow[t]{2}{*}{ Clinical Characteristics } & \multicolumn{2}{|c|}{ Expression of ARLI 4 in LUAD } & \multirow[t]{2}{*}{$\mathbf{P}$} & \multicolumn{2}{|c|}{ Expression of ARLI4 in LUSC } & \multirow[t]{2}{*}{$\mathbf{P}$} \\
\hline & Low & High & & Low & High & \\
\hline Number & 267 & 268 & & 251 & 251 & \\
\hline Age (years) & & & 0.596 & & & 0.003 \\
\hline$\leq 65$ & $132(25.6 \%)$ & $123(23.8 \%)$ & & III (22.5\%) & $80(16.2 \%)$ & \\
\hline$>65$ & $128(24.8 \%)$ & $133(25.8 \%)$ & & $133(27 \%)$ & $169(34.3 \%)$ & \\
\hline Gender & & & 0.280 & & & 0.416 \\
\hline Female & I 36 (25.4\%) & I50 (28\%) & & 70 (I3.9\%) & $61(12.2 \%)$ & \\
\hline Male & $|3|(24.5 \%)$ & $118(22.1 \%)$ & & $181(36.1 \%)$ & $190(37.8 \%)$ & \\
\hline Race & & & 0.358 & & & 0.135 \\
\hline Asian & $3(0.6 \%)$ & $4(0.9 \%)$ & & $3(0.8 \%)$ & $6(1.5 \%)$ & \\
\hline Black or African American & $32(6.8 \%)$ & $23(4.9 \%)$ & & $10(2.6 \%)$ & $20(5.1 \%)$ & \\
\hline White & $196(41.9 \%)$ & $210(44.9 \%)$ & & $176(45.2 \%)$ & $174(44.7 \%)$ & \\
\hline Smoker & & & 0.121 & & & 0.797 \\
\hline No & $31(6 \%)$ & $44(8.4 \%)$ & & $10(2 \%)$ & $8(1.6 \%)$ & \\
\hline Yes & 231 (44.3\%) & $215(41.3 \%)$ & & $234(47.8 \%)$ & $238(48.6 \%)$ & \\
\hline T stage & & & 0.227 & & & 0.867 \\
\hline TI & $85(16 \%)$ & 90 (16.9\%) & & $60(12 \%)$ & $54(10.8 \%)$ & \\
\hline $\mathrm{T} 2$ & 154 (28.9\%) & 135 (25.4\%) & & 146 (29.1\%) & I 48 (29.5\%) & \\
\hline T3 & $19(3.6 \%)$ & 30 (5.6\%) & & $33(6.6 \%)$ & $38(7.6 \%)$ & \\
\hline $\mathrm{T} 4$ & $8(1.5 \%)$ & II (2.1\%) & & $12(2.4 \%)$ & II (2.2\%) & \\
\hline N stage & & & 0.385 & & & 0.009 \\
\hline No & I8I (34.9\%) & 167 (32.2\%) & & 146 (29.4\%) & $174(35.1 \%)$ & \\
\hline $\mathrm{NI}$ & 45 (8.7\%) & 50 (9.6\%) & & $80(16.1 \%)$ & $5 \mathrm{I}(10.3 \%)$ & \\
\hline N2 & 31 (6\%) & 43 (8.3\%) & & 22 (4.4\%) & $18(3.6 \%)$ & \\
\hline N3 & I (0.2\%) & I (0.2\%) & & $\mathrm{I}(0.2 \%)$ & $4(0.8 \%)$ & \\
\hline M stage & & & 0.979 & & & 0.273 \\
\hline Mo & 179 (46.4\%) & I 82 (47.2\%) & & $213(50.8 \%)$ & 199 (47.5\%) & \\
\hline MI & $13(3.4 \%)$ & $12(3.1 \%)$ & & $2(0.5 \%)$ & $5(1.2 \%)$ & \\
\hline Pathologic stage & & & 0.314 & & & 0.330 \\
\hline $\mathrm{I}$ & I 55 (29.4\%) & $139(26.4 \%)$ & & $115(23.1 \%)$ & $130(26.1 \%)$ & \\
\hline II & $58(11 \%)$ & 65 (12.3\%) & & $86(17.3 \%)$ & $76(15.3 \%)$ & \\
\hline III & 35 (6.6\%) & 49 (9.3\%) & & $46(9.2 \%)$ & $38(7.6 \%)$ & \\
\hline IV & $13(2.5 \%)$ & $13(2.5 \%)$ & & $2(0.4 \%)$ & $5(1 \%)$ & \\
\hline Residual tumor & & & 0.017 & & & 0.708 \\
\hline Ro & $178(47.8 \%)$ & $177(47.6 \%)$ & & 205 (49.4\%) & $194(46.7 \%)$ & \\
\hline $\mathrm{RI}$ & $10(2.7 \%)$ & $3(0.8 \%)$ & & $6(1.4 \%)$ & $6(1.4 \%)$ & \\
\hline $\mathrm{R} 2$ & $0(0 \%)$ & 4 (I.1\%) & & I (0.2\%) & $3(0.7 \%)$ & \\
\hline
\end{tabular}


None of the selected patients had any history of other malignant tumors, immune dysfunction, and preoperative radiotherapy or chemotherapy. Two pathologists provided a diagnosis and defined histological typing and tumor staging according to the 8th edition of the tumor, node, metastasis (TNM) staging system for lung cancer. ${ }^{18}$ Follow-up data for patients expired on December 30, 2020 , or the date of death. All the patients we registered provided the following clinical and pathologic information: age, sex, operative methods, smoking history, tumor size, pathological type, pathological stage, $\mathrm{T}$ stage, $\mathrm{N}$ stage, $\mathrm{M}$ stage, tumor differentiation, treatment after surgery, follow-up status, tumor recurrence and progression, overall survival (OS) time, and progression-free survival time (PFS) (Table 2). This study was approved by the Medical Ethics Committee.

Table 2 Correlation Between ARLI4 Expression and Clinicopathological Characteristics in I20 Patients with NSCLC

\begin{tabular}{|c|c|c|c|c|}
\hline \multirow[t]{2}{*}{ Characteristics } & \multirow[t]{2}{*}{ All Patients $(n=120)$} & \multicolumn{2}{|c|}{ ARLI4 Expression } & \multirow[t]{2}{*}{$\mathbf{P}$} \\
\hline & & Low $(n=74)$ & High $(n=46)$ & \\
\hline Age (years) & & & & 0.222 \\
\hline$<55$ & 44 & 24 & 20 & \\
\hline$\geq 55$ & 76 & 50 & 26 & \\
\hline Gender & & & & 0.288 \\
\hline Male & 80 & 52 & 28 & \\
\hline Female & 40 & 22 & 18 & \\
\hline Smoke & & & & 0.125 \\
\hline Never & 42 & 22 & 20 & \\
\hline Ever & 78 & 52 & 26 & \\
\hline Type of operation & & & & 0.125 \\
\hline Lobectomy & 111 & 68 & 43 & \\
\hline Sleeve lobectomy & 2 & 0 & 2 & \\
\hline Pneumonectomy & 4 & 4 & 0 & \\
\hline Wedge resection or segmentectomy & 3 & 2 & 1 & \\
\hline Maximum diameter of tumor & & & & 0.98 \\
\hline$\leq 4 \mathrm{~cm}$ & 68 & 42 & 26 & \\
\hline$>4 \mathrm{~cm}$ & 52 & 32 & 20 & \\
\hline Pathological type & & & & 0.253 \\
\hline Adenocarcinoma & 56 & 34 & 22 & \\
\hline Squamous cell carcinoma & 52 & 30 & 22 & \\
\hline Other & 12 & 10 & 2 & \\
\hline T stage & & & & 0.345 \\
\hline TI & 51 & 28 & 23 & \\
\hline $\mathrm{T} 2$ & 42 & 30 & 12 & \\
\hline T3 & 17 & 11 & 6 & \\
\hline $\mathrm{T} 4$ & 10 & 5 & 5 & \\
\hline N stage & & & & 0.944 \\
\hline No & 88 & 55 & 33 & \\
\hline $\mathrm{NI}$ & 14 & 9 & 5 & \\
\hline N2 & 16 & 9 & 7 & \\
\hline N3 & 2 & 1 & 1 & \\
\hline$M$ stage & & & & 0.625 \\
\hline Mo & 116 & 72 & 44 & \\
\hline MI & 4 & 2 & 2 & \\
\hline
\end{tabular}

(Continued) 
Table 2 (Continued).

\begin{tabular}{|c|c|c|c|c|}
\hline \multirow[t]{2}{*}{ Characteristics } & \multirow[t]{2}{*}{ All Patients $(n=120)$} & \multicolumn{2}{|c|}{ ARLI 4 Expression } & \multirow[t]{2}{*}{$\mathbf{P}$} \\
\hline & & Low $(n=74)$ & High $(n=46)$ & \\
\hline Pathologic stage & & & & 0.488 \\
\hline I & 66 & 39 & 27 & \\
\hline II & 27 & 20 & 7 & \\
\hline III & 23 & 13 & 10 & \\
\hline IV & 4 & 2 & 2 & \\
\hline Differentiation & & & & 0.011 \\
\hline Well & 25 & 18 & 7 & \\
\hline Moderate & 50 & 36 & 14 & \\
\hline Poor & 45 & 20 & 25 & \\
\hline Treatment after surgery & & & & 0.155 \\
\hline None & 72 & 43 & 29 & \\
\hline Chemotherapy & 20 & 16 & 4 & \\
\hline Targeted therapy & 28 & 15 & 13 & \\
\hline
\end{tabular}

\section{IHC}

The experiments were performed similar to previously described. ${ }^{19,20}$ After deparaffinization in xylene and rehydration in a graded ethanol series, antigen retrieval was performed in citrate-buffered solution ( $\mathrm{pH}$ 6.0). To block endogenous peroxidase, $10 \%$ normal goat serum was used for blocking. They were incubated overnight at $4{ }^{\circ} \mathrm{C}$ with an anti-ARL14 antibody. After washing with phosphate-buffered saline (PBS), they were incubated for one hour at room temperature with a secondary antibody. They were stained with 3, 3-diaminobenzidine tetrahydrochloride (DAB), counterstained with hematoxylin, dehydrated, cleared, and evaluated. Cytoplasmic staining was identified as positive for ARL14 expression in tumors.

Two independent pathologists evaluated ARL14 expression under light microscopy at $400 \times$ magnification, according to the following criteria: (i) intensity of staining: 0 (negative staining), 1 (mild staining), 2 (moderate staining), and 3 (intense staining); (ii) percentage of immunopositive cells (five randomly selected visual fields at $400 \times$ magnification of each slide): 0 (0\%), 1 (1-25\%), 2 (26-50\%), 3 (51-75\%), 4 (76-100\%). Final scores ranging from 0 to 12 were obtained by multiplying the above two scoring systems. We defined 0-2 points as negative expression, while 3-6 points as low ARL14 expression and 7-12 points as high ARL14 expression. ${ }^{21}$

\section{Enrichment Analyses}

Enrichment analyses were performed for LUAD and LUSC. Correlation analysis between ARL14 and other mRNAs in
LUAD and LUSC was performed using TCGA data, and the Pearson correlation coefficient was calculated. The top 1200 genes most associated with ARL14 were selected for enrichment analysis. We used the R clusterProfiler package for GO and KEGG analysis. ${ }^{22}$ GSEA was also performed using the gseGO, gseKEGG, and gsePathway functions of the R package "clusterProfiler". ${ }^{22}|\mathrm{NES}|>1$, adjusted $\mathrm{P}<0.05$, and FDR $<0.25$ were considered statistically significant.

\section{Immune Cell Infiltration}

The immune infiltration analysis of LUAD and LUSC was performed using ssGSEA (within GSVA package). ${ }^{23}$ We also evaluated the relationship between ARL14 expression and the abundance of 24 immune cell types. We also evaluated the relationship Using the Spearman and Wilcoxon rank sum tests, $\mathrm{P}<0.05$ was regarded as statistically significant.

\section{Statistical Analysis}

All statistical analyses of TCGA data were conducted using R. The expression levels of ARL14 in patients were determined using the ggplot2 package. ${ }^{24}$ The SPSS statistical software program and GraphPad Prism were used to analyze the data and generate figures for 120 patients with NSCLC. Wilcoxon signed-rank test, Chi-square test, and Fisher's exact test were performed to analyze the association between clinical features and ARL14 expression. Kaplan-Meier analysis was used to evaluate the prognostic value of ARL14 in NSCLC using a Log rank test. A Cox proportional-hazards regression model was used for the univariate and multivariate 
analyses of survival. Factors that showed statistical significance in univariate analysis $(\mathrm{P}<0.05)$ were included in multivariate analysis to identify independent risk factors. A nomogram was constructed to predict 1-, 3- and 5-year NSCLC overall survival by combining the expression value of ARL14 with clinical variables. Statistical significance was set at $\mathrm{P}<0.05$.

\section{Results}

High ARLI 4 Expression in LUAD and LUSC A total of 535 patients with LUAD and 502 patients with LUSC presenting the required clinical features were acquired from TCGA data. The detailed clinical features are shown in Table 1. Among the participants, 249 patients with LUAD were male (46.6\%), and 286 were female (53.4\%), while 371 patients with LUSC were male (73.9\%), and 131 were female
(26.1\%). The median ages of these patients were 66 and 68 years, respectively. In terms of the LUAD stage, 294 patients were at stage I (55.8\%), 123 patients were at stage II (23.3\%), 84 patients were at stage III (15.9\%), and 26 patients were at stage IV (5.0\%). In LUSC, there were 245 (49.2\%) patients at stage I, $162(32.6 \%)$ at stage II, $84(16.8 \%)$ at stage III, and seven $(1.4 \%)$ at stage IV. Most participants smoked, including patients with LUAD 446 (85.6\%) and LUSC 472 (96.4\%).

To assess the status of ARL14 expression in patients with NSCLC, we compared the expression levels to those in normal lung tissues. The results demonstrated that ARL14 gene expression levels in both LUAD and LUSC tissues were significantly higher than those in normal tissues $(\mathrm{P}<0.001$; Figure $1 \mathrm{~A}$ and $\mathrm{C})$. The results were verified in LUAD and LUSC tissues and paired normal lung tissues $(\mathrm{P}<0.001$; Figure $1 \mathrm{~B}$ and $\mathrm{D})$.
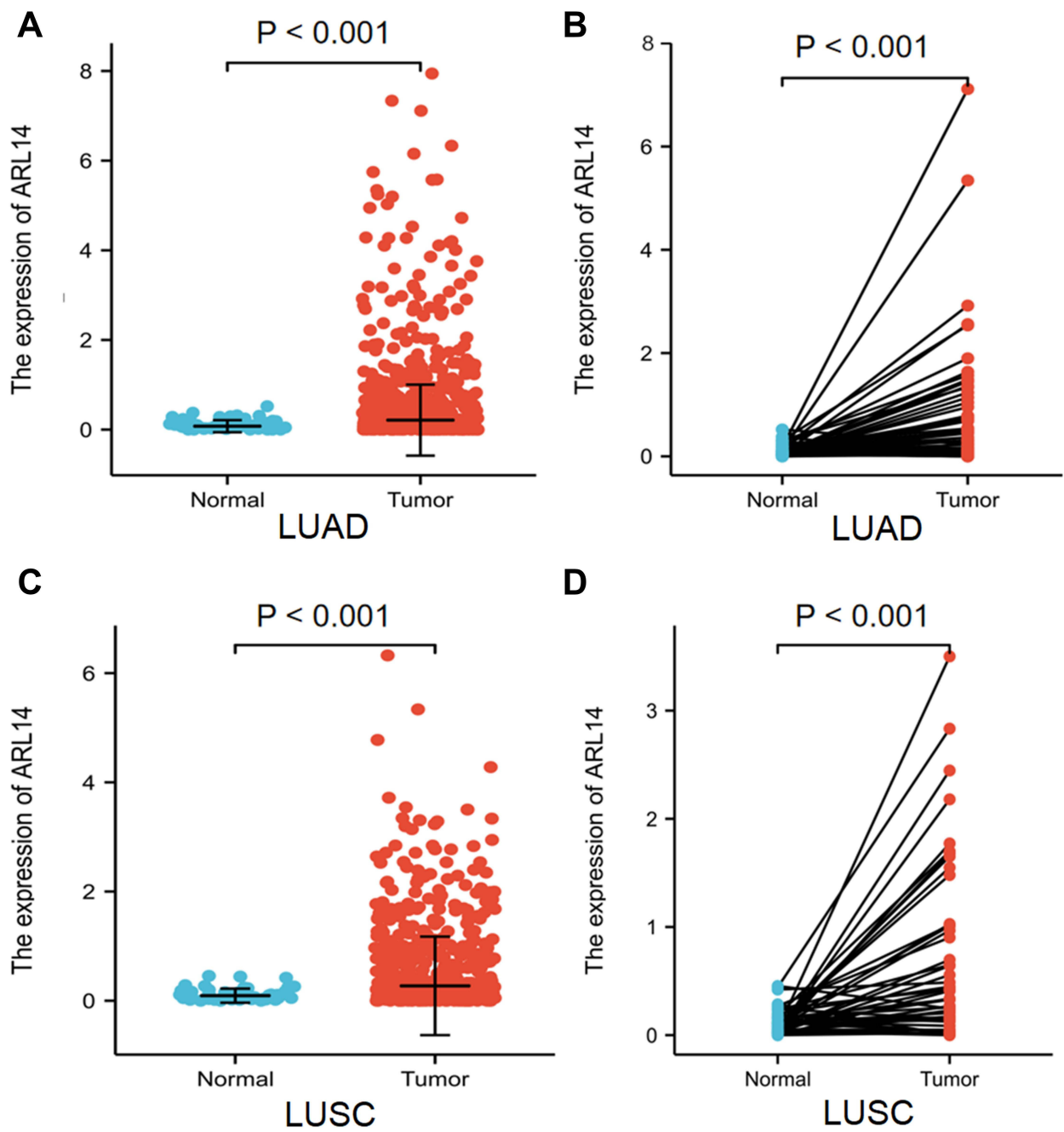

Figure I ARLI4 expression in LUAD and LUSC. (A) ARLI4 expression in LUAD tissues and normal samples. (B) ARLI4 expression in LUAD tissues and paired normal samples. (C) ARLI4 expression in LUSC tissues and normal samples. (D) ARLI4 expression in LUSC tissues and paired normal samples. 

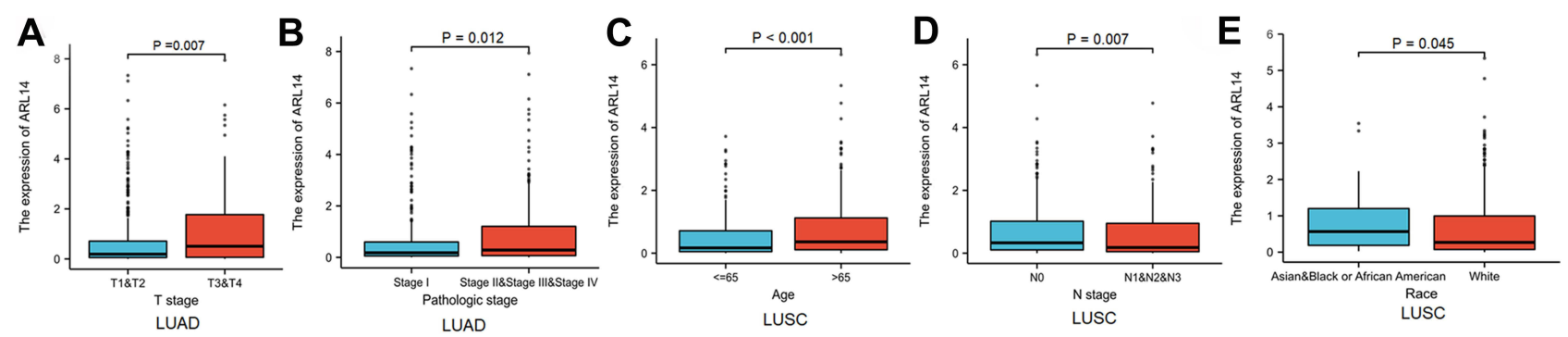

Figure 2 Correlation between ARLI4 expression and clinicopathologic features in patients with LUAD and LUSC in TCGA database (P< 0.05$)$. (A and B) The correlation between ARLI4 expression and T stage (A) and pathologic stage (B) of patients with LUAD. (C-E) The correlation between the expression of ARLI4 and age (C), N stage (D) and race (E) of patients with LUSC.

Furthermore, different ARL14 expression was observed in groups based on age, sex, race, smoking history, $\mathrm{T}$ stage, $\mathrm{N}$ stage, $\mathrm{M}$ stage, and pathologic stage (Figures 2, $\underline{\mathrm{S} 1 \mathrm{~A}-\mathrm{K}}$ ). The ARL14 expression in patients LUAD in T3/T4 stage was higher than that in patients in $\mathrm{T} 1 / \mathrm{T} 2$ stage $(\mathrm{P}=0.007$; Figure 2A), while there was no significant difference in patients with LUSC ( $\mathrm{P}=0.911$; Figure S1I). Patients with LUAD who were in the high pathologic stage had higher ARL14 expression than patients in the low pathologic stage $(\mathrm{P}=0.012$; Figure 2B). However, high expression of ARL14 was not significantly correlated with other clinical features in LUAD (Figure S1A-F). High expression of ARL14 was significantly correlated with age $(\mathrm{P}<0.001)$, race $(\mathrm{P}=0.045)$, and $\mathrm{N}$ stage $(\mathrm{P}=0.007)$ in LUSC, as shown in Figure $2 \mathrm{C}-\mathrm{E}$.

\section{Correlation Between Clinical Characteristics and ARLI4 Expression of LUAD and LUSC}

The associations between clinical characteristics and ARL14 expression in LUAD and LUSC were investigated. The patients were divided into high- and low-expression groups based on ARL14 expression. The Chi-square test or Fisher's exact test revealed that ARL14 expression was associated with residual tumor $(\mathrm{P}=0.017)$ in LUAD, while it was associated with age $(\mathrm{P}=0.003)$ and $\mathrm{N}$ stage $(\mathrm{P}=0.009)$ in LUSC (Table 1).

\section{High ARL 4 Expression is an Independent Risk Factor for OS in Patients with LUAD and LUSC}

The association between ARL14 expression and OS in patients with LUAD and LUSC was evaluated by KaplanMeier analysis, which indicated that high expression of ARL14 was positively correlated with the poor OS of patients with LUAD $(\mathrm{P}=0.03$; Figure $3 \mathrm{~A})$ and LUSC $(\mathrm{P}=0.022$; Figure $3 \mathrm{G})$. Subgroup analysis indicated that ARL14 overexpression significantly affected the OS in LUAD in T3/T4 stage $(\mathrm{P}=0.005), \mathrm{N} 1 / \mathrm{N} 2 / \mathrm{N} 3(\mathrm{P}=0.048), \mathrm{M} 0(\mathrm{P}=0.033)$, female ( $\mathrm{P}=0.023)$, and $\mathrm{R} 0(\mathrm{P}=0.048)$ (Figure $3 \mathrm{~B}-\mathrm{F})$. In LUSC, ARL14 overexpression significantly affected the OS in patients aged $\leq 65$ years $(\mathrm{P}=0.025)$, smokers $(\mathrm{P}=0.029)$,

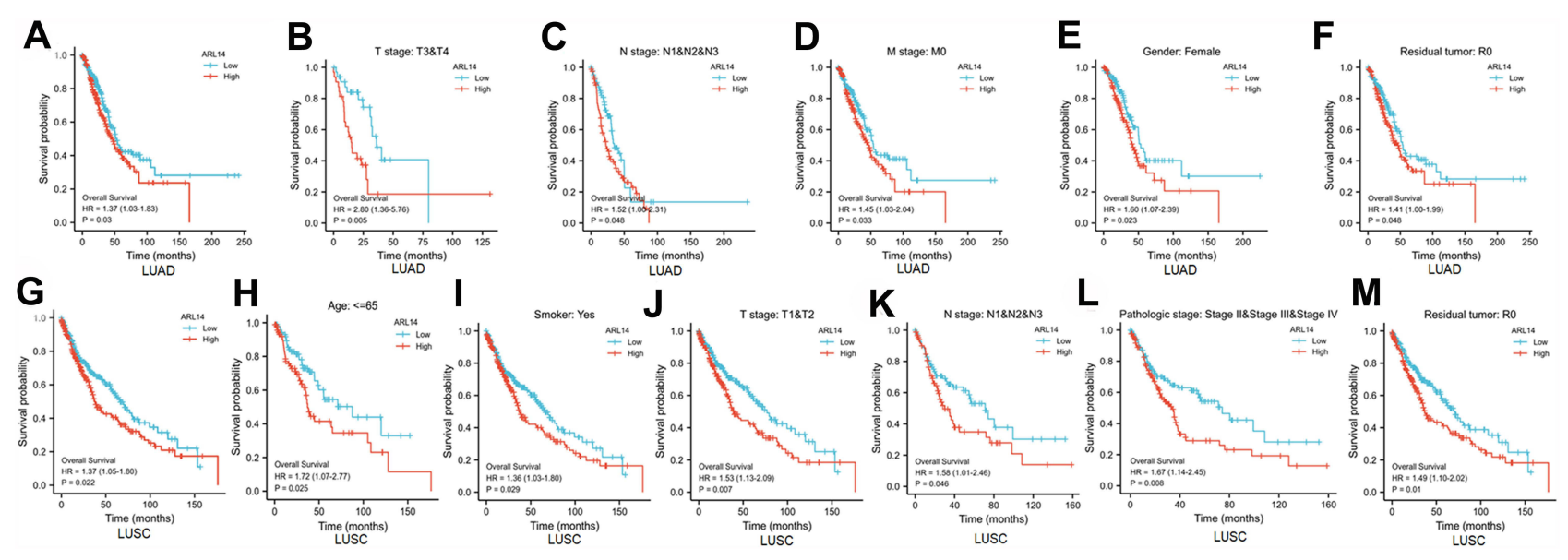

Figure 3 Kaplan-Meier curve for OS in LUAD and LUSC. (A) Kaplan-Meier analysis for ARLI4 in LUAD; (B-F) Subgroup analysis for T3/T4, NI/N2/N3, M0, female, and R0 in LUAD. (G) Kaplan-Meier analysis for ARLI4 in LUSC; (H-M) Subgroup analysis for age $\leq 65$ years, smoker, TI/T2, NI/N2/N3, pathologic stage II/III/IV, and R0 in LUSC. 
in $\mathrm{T} 1 / \mathrm{T} 2$ stage $(\mathrm{P}=0.007), \mathrm{N} 1 / \mathrm{N} 2 / \mathrm{N} 3(\mathrm{P}=0.046)$, pathologic stage II/III/IV $(\mathrm{P}=0.008)$, and R0 $(\mathrm{P}=0.01)$ (Figure $3 \mathrm{H}-\mathrm{M})$.

Univariate Cox regression analysis revealed that higher ARL14 expression, advanced clinical stage, more residual tumor, distant metastasis, and positive lymph nodes were correlated with OS, while multivariate analysis showed that ARL14 expression was an independent risk factor for OS in patients with LUAD (Table 3). Similar results were found in patients with LUSC (Table 3). Then, we constructed prognostic nomograms to anticipate the individuals' survival probability by weighing $\mathrm{T}$ stage, $\mathrm{N}$ stage, $\mathrm{M}$ stage, pathologic stage, residual tumor, and ARL14 expression in LUAD and LUSC respectively. A C-index was used to appraise the nomogram's predictive discrimination. In our prognostic nomogram, the C-index was 0.676 in LUAD and 0.594 in LUSC (Figure 4).

\section{ARLI4 Overexpression in NSCLC Tissues Was Shown Using IHC}

IHC was performed on 120 human NSCLC tissues and 120 adjacent normal lung tissues. The clinicopathological factors of the 120 patients with NSCLC are illustrated in Table 2. Forty female and 80 male patients were included in the study. Regarding smoking history, 78 patients had a history of smoking, and 42 patients were non-smokers. Regarding the pathologic stage, 66 patients were at stage I, 27 patients were at stage II, 23 patients were at stage III, and four patients were at stage IV. Surgical operations included lobectomy in 111 patients, pneumonectomy in four, sleeve lobectomy in two, and wedge resection or segmentectomy in three. IHC staining of ARL14 showed high expression levels in 46 cases and low expression levels in 74 cases.

ARL14 expression was upregulated in NSCLC tissues compared to adjacent normal lung tissues $(\mathrm{P}<0.05$; Figure 5A). High and low ARL14 expression levels in NSCLC tissues and adjacent normal lung tissues detected by immunohistochemical staining are shown in Figure 5B-D. This result is consistent with the conclusion obtained using TCGA database.

\section{Correlation Between ARLI4 Expression and Clinicopathologic Characteristics in NSCLC Tissue Samples}

Compared to the adjacent normal lung tissues in which ARL14 was undetectable or found to be only expressed at low levels, ARL14 was overexpressed in NSCLC tissues. The association between ARL14 expression and diverse clinicopathological factors is shown in Table 2. High levels of ARL14 were significantly associated with differentiation $(\mathrm{P}=$ 0.011). However, no significant associations were observed between ARL14 and sex $(\mathrm{P}=0.288)$, age $(\mathrm{P}=0.222)$, smoking history $(\mathrm{P}=0.125)$, type of operation $(\mathrm{P}=0.125)$, tumor size $(\mathrm{P}=0.98)$, histological classification $(\mathrm{P}=0.253)$, $T$ classification $(\mathrm{P}=0.345), \mathrm{N}$ classification $(\mathrm{P}=0.944)$, $\mathrm{M}$ classification $(\mathrm{P}=0.625)$, and clinical stage $(\mathrm{P}=0.488)$.

\section{High ARLI4 Protein Levels Predicted Poor Survival of 120 Patients with NSCLC}

Cox regression analysis was used to determine whether ARL14 could serve as a risk factor. As shown in Table 4,

Table 3 Correlations Between Overall Survival and Expression of ARLI4 Analyzed by Univariate and Multivariate Cox Regression in LUAD and LUSC

\begin{tabular}{|c|c|c|c|c|}
\hline \multirow[t]{2}{*}{ Clinical Characteristics } & \multicolumn{2}{|c|}{ Univariate Analysis } & \multicolumn{2}{|c|}{ Multivariate Analysis } \\
\hline & Hazard Ratio $(95 \% \mathrm{Cl})$ & $\mathbf{P}$ & Hazard Ratio (95\% Cl) & $\mathbf{P}$ \\
\hline \multicolumn{5}{|l|}{ LUAD } \\
\hline ARLI4 (High vs Low) & $1.375(1.031-1.834)$ & 0.03 & $1.496(1.029-2.173)$ & 0.035 \\
\hline T stage (T3 \& T4 vs TI \& T2) & $1.728(1.229-2.43 \mathrm{I})$ & 0.002 & $1.664(1.027-2.695)$ & 0.038 \\
\hline$N$ stage (NI \& N2 \& N3 vs N0) & $2.321(1.631-3.303)$ & $<0.001$ & $1.503(0.625-3.616)$ & 0.363 \\
\hline M stage (MI vs M0) & $2.136(1.248-3.653)$ & 0.006 & $0.937(0.364-2.410)$ & 0.892 \\
\hline Pathologic stage (Stage II \& Stage III \& Stage IV vs Stage I) & $2.664(1.960-3.621)$ & $<0.001$ & $\mathrm{I} .802(0.76 \mathrm{I}-4.268)$ & 0.18 \\
\hline Residual tumor (RI \& R2 vs R0) & $3.879(2.169-6.936)$ & $<0.001$ & $3.317(1.558-7.062)$ & 0.002 \\
\hline \multicolumn{5}{|l|}{ LUSC } \\
\hline ARLI4 (High vs Low) & $1.373(1.046-1.802)$ & 0.022 & $1.341(1.017-1.770)$ & 0.038 \\
\hline$T$ stage ( $T 3$ \& $T 4$ vs $T 1$ \& $T 2$ ) & $1.658(1.200-2.291)$ & 0.002 & $1.457(0.994-2.135)$ & 0.054 \\
\hline Pathologic stage (Stage II \& Stage III \& Stage IV vs Stage I) & $1.321(1.006-1.735)$ & 0.046 & $1.170(0.85 I-1.609)$ & 0.334 \\
\hline
\end{tabular}




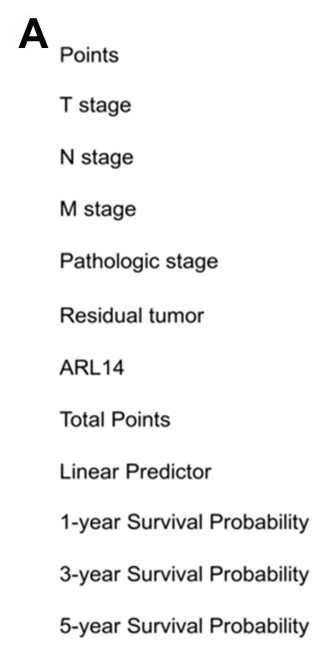

B

Points
T stage
N stage
M stage
Pathologic stage
Residual tumor
ARL14
Total Points
Linear Predictor
1-year Survival Probability
3-year Survival Probability
5-year Survival Probability
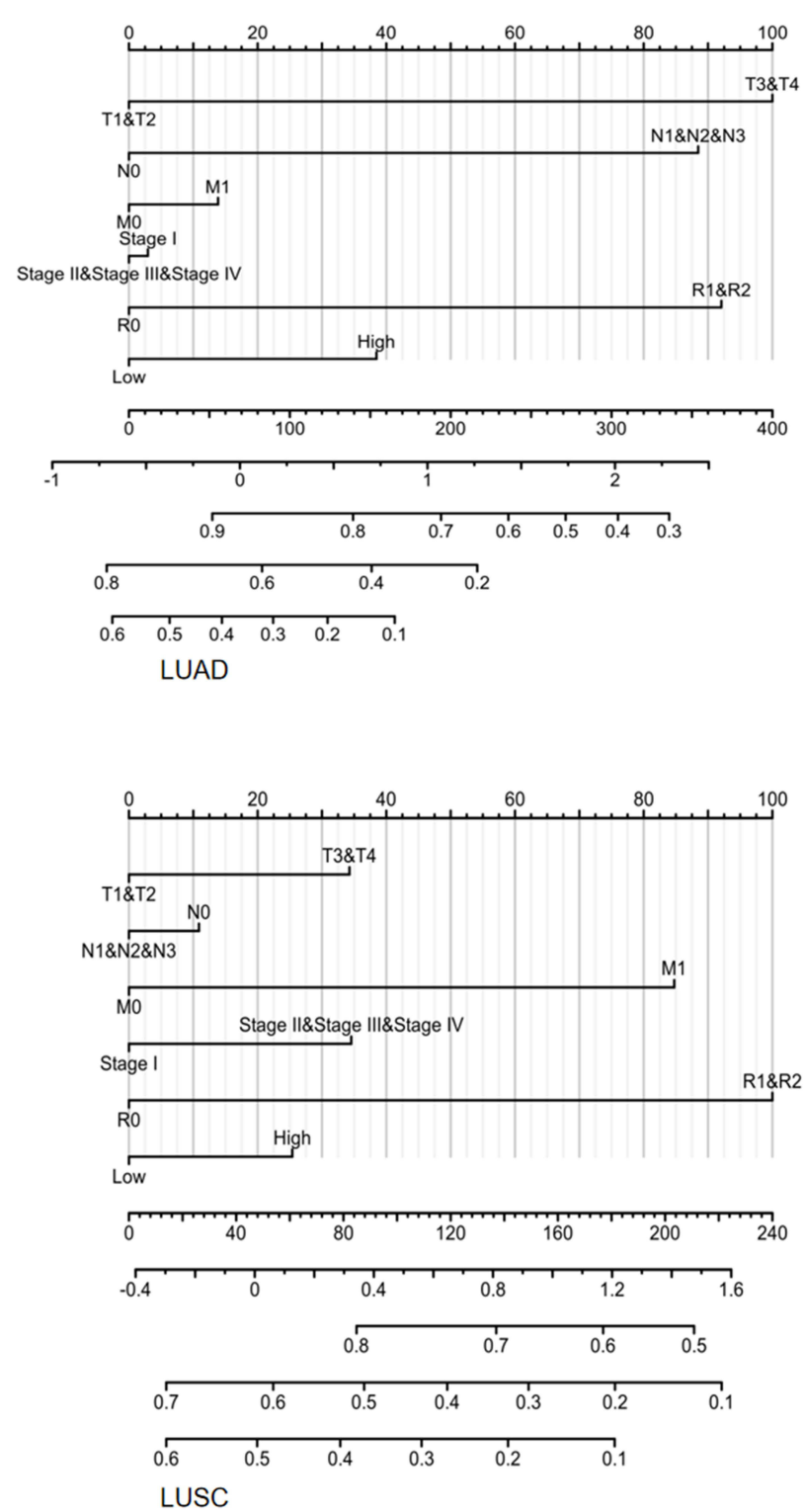

Figure 4 Nomogram for predicting probability of patients with I-, 3- and 5-year OS. (A) Nomogram for predicting probability of patients with LUAD in TCGA database. (B) Nomogram for predicting probability of patients with LUSC in TCGA database.

high expression of ARL14 was associated with a significantly increased risk of death in patients with NSCLC (hazard ratio (HR) 0.213, 95\% confidence interval (CI) $0.125-0.364$; $\mathrm{P}<0.001$ ) compared to those with low ARL14 expression by univariate Cox regression analyses. Furthermore, the results of univariate analysis demonstrated that age (HR 2.388, 95\% CI 1.427-3.996; $\mathrm{P}=0.001$ ), differentiation (HR 0.558 , 95\% CI 0.382 $0.814 ; \mathrm{P}=0.002), \mathrm{M}$ classification ( $\mathrm{HR} 5.611,95 \% \mathrm{CI}$ 1.952-16.126; $\mathrm{P}=0.001$ ), and clinical stage (HR 1.419, $95 \%$ CI 1.064-1.894; $\mathrm{P}=0.017$ ) were the key factors leading to poorer OS in patients with NSCLC. Multivariate Cox regression analysis showed that ARL14 expression (HR 0.248, 95\% CI 0.140-0.440; P < 0.001) was an independent prognostic factor for OS (Table 4). The results of univariate analysis of PFS were similar to those observed in OS, with ARL14 expression (HR 0.23, 95\% CI 0.135-0.392; P < 0.001), age (HR 2.529, 95\% CI 1.509-4.24; $\mathrm{P}<0.001$ ), differentiation (HR 0.574, 95\% CI 0.394-0.835; $\mathrm{P}=0.004$ ), $\mathrm{M}$ classification (HR 4.177, 95\% CI 1.458-11.973; $\mathrm{P}=0.008)$, and clinical stage (HR 1.403, 95\% CI 1.054-1.866; $\mathrm{P}=0.02$ ). In multivariate analysis, age (HR 1.929, 95\% CI 1.127-3.302; $\mathrm{P}=$ 0.017 ) and ARL14 expression (HR 0.279, 95\% CI 0.158-0.491; $\mathrm{P}<0.001)$ were independent predictors of PFS, as shown in Table 4. 
A

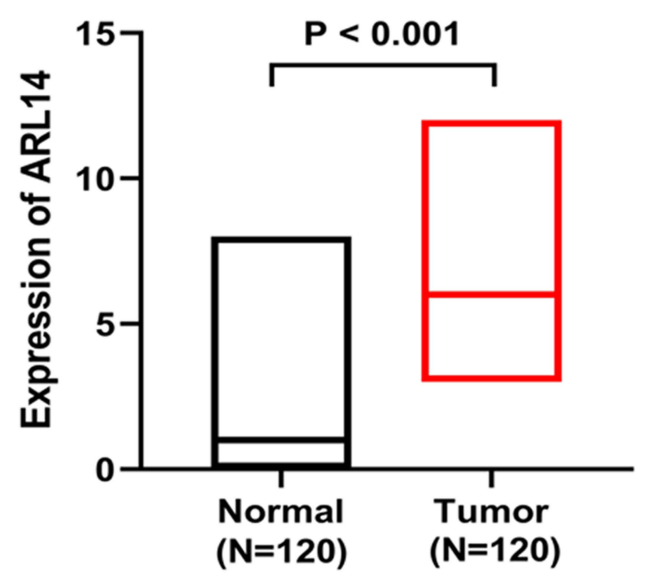

C

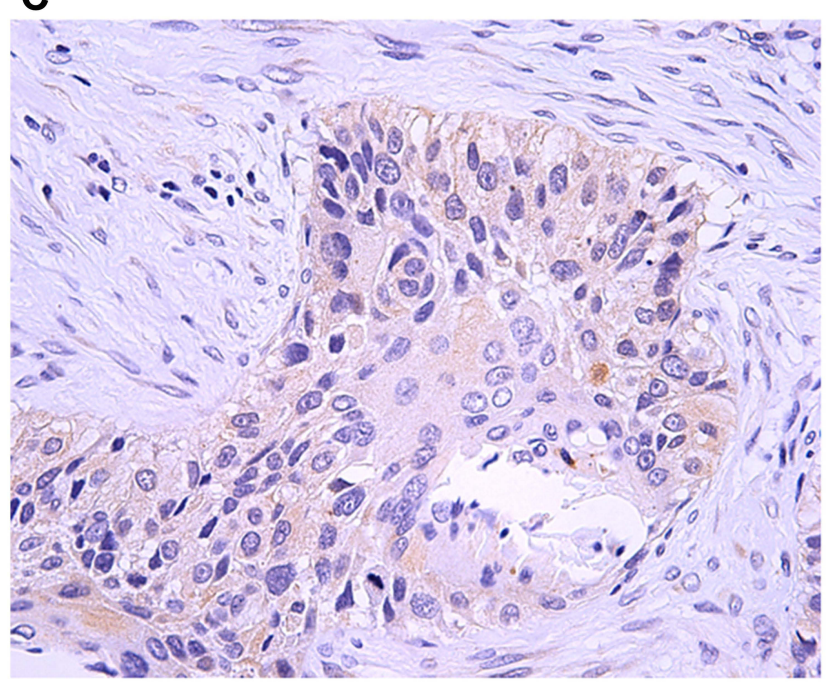

E

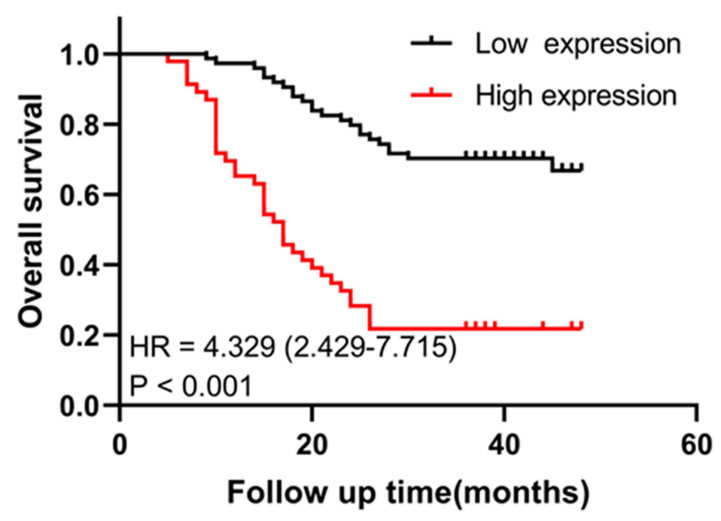

B

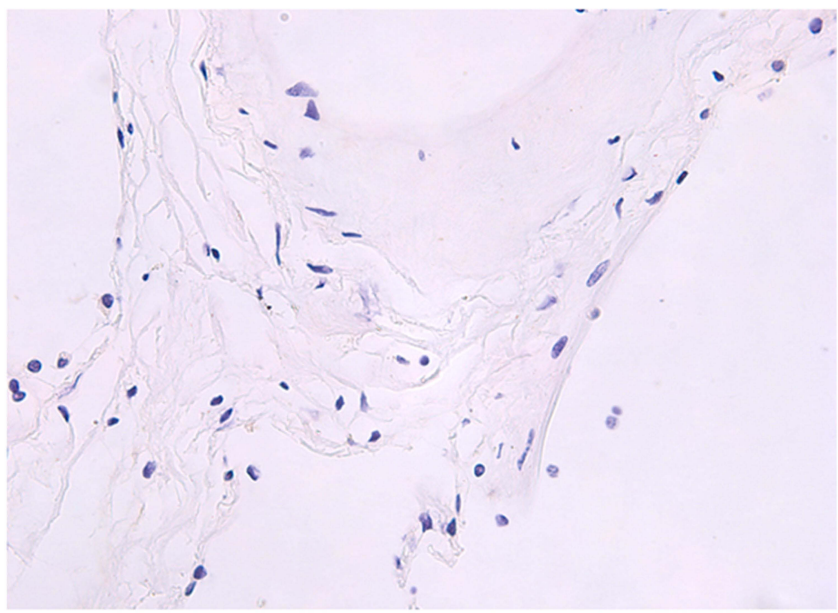

D

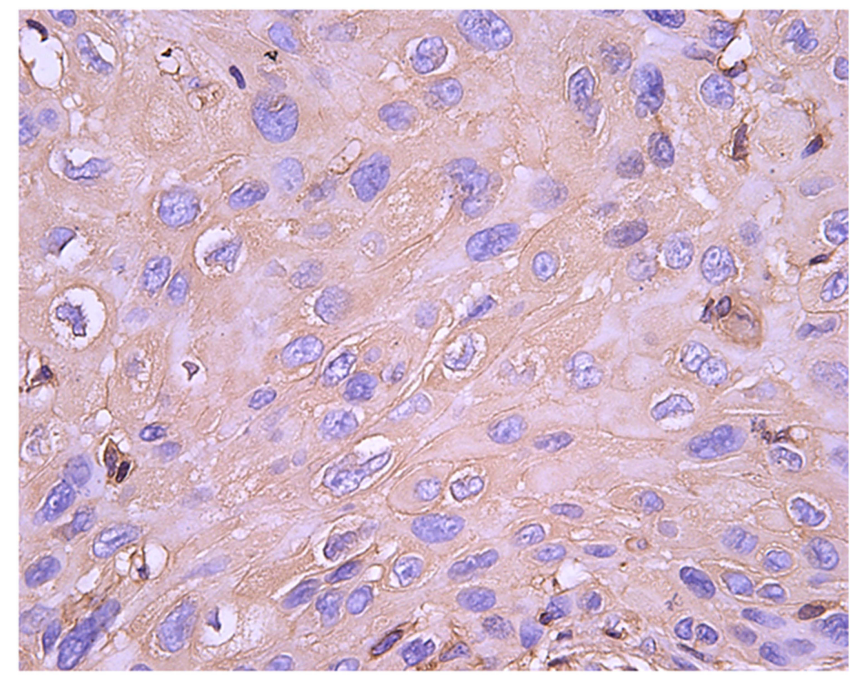

F

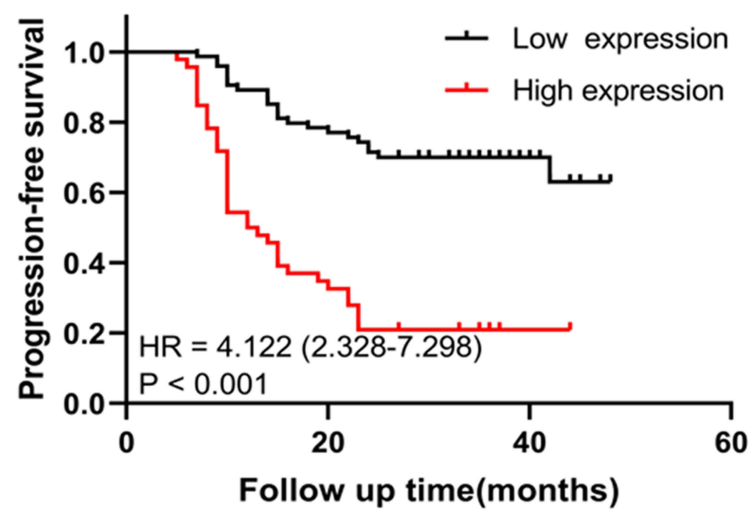

Figure 5 IHC staining and survival analysis of ARLI4 in human NSCLC. (A) ARLI4 expression of IHC on I20 human NSCLC tissues and I20 adjacent normal lung tissues. (B) Immunohistochemical staining for ARLI4 expression in adjacent normal lung tissues $(\times 400)$. (C) Immunohistochemical staining for low ARLI4 expression in NSCLC tissues (×400). (D) Immunohistochemical staining for high ARLI4 expression in NSCLC tissues ( $\times 400)$. (E) Kaplan-Meier plots of OS according to ARLI4 expression. (F) Kaplan-Meier plots of PFS according to ARLI4 expression. 


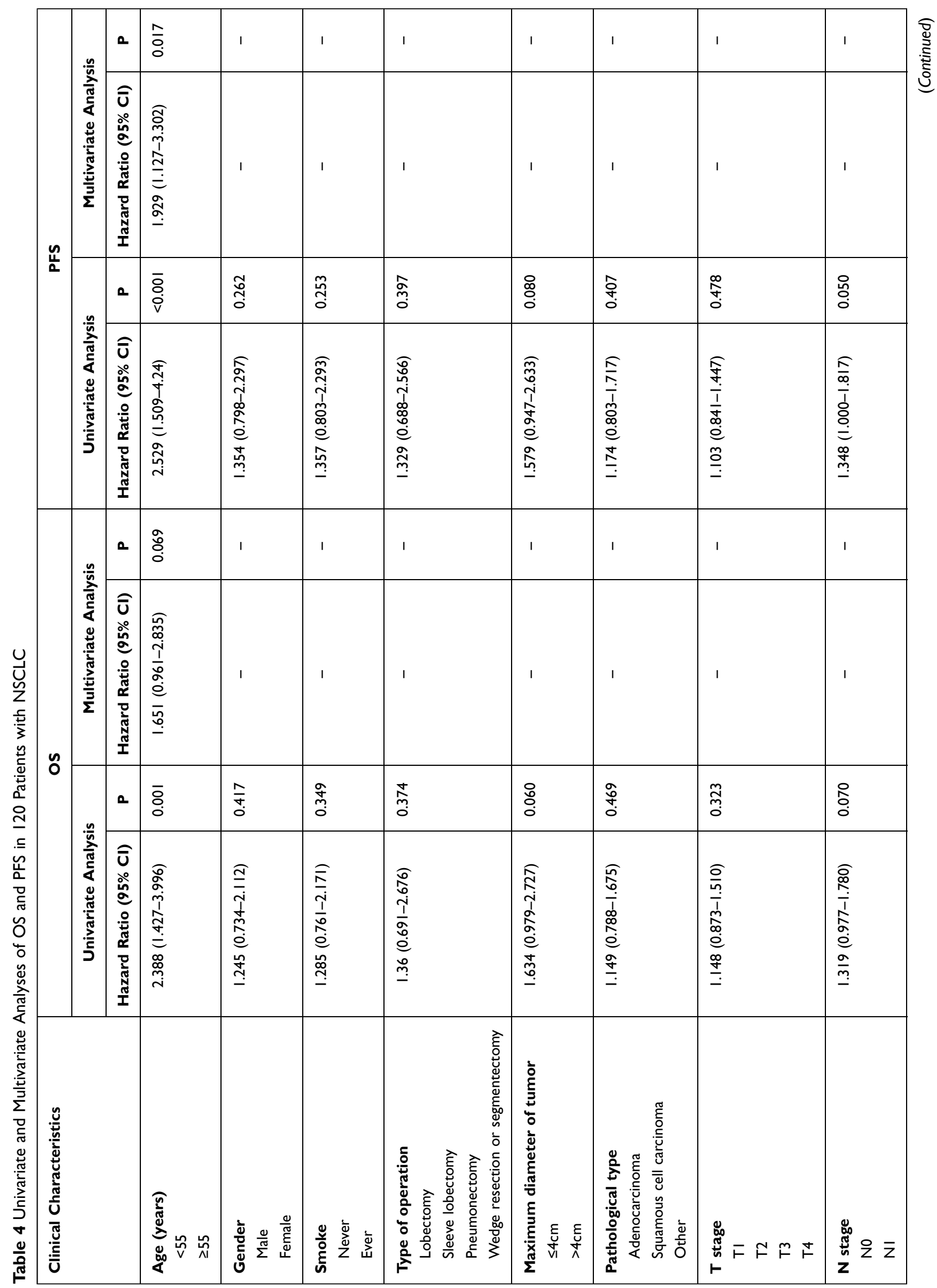




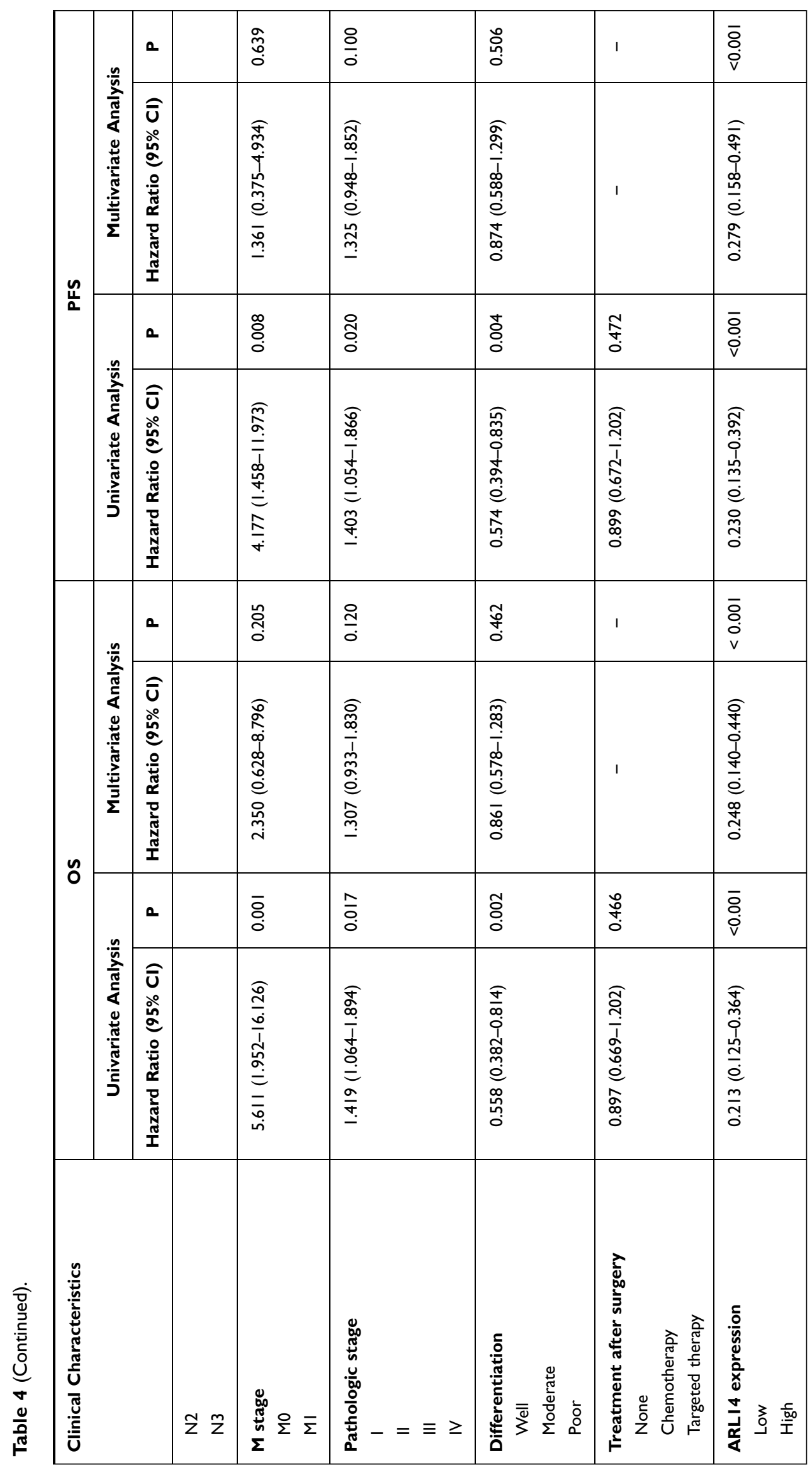


Kaplan-Meier survival curves indicated that the OS (HR 4.329, 95\% CI 2.429-7.715; P < 0.001) and PFS (HR 4.122, 95\% CI 2.328-7.298; $\mathrm{P}<0.001$ ) of patients with high expression of ARL14 were significantly shorter than those of patients with low ARL14 expression (Figure 5E and F). Collectively, these results demonstrate that overexpression of ARL14 in patients with NSCLC correlated with poor survival. These results are similar to those of TCGA cohort.

\section{Correlation and Enrichment Analyses}

We performed a correlation analysis between ARL14 and other genes in LUAD and LUSC using TCGA data to predict the function of ARL14, with the top 50 genes most positively and the top 50 genes most negatively associated with ARL14 (Figure 6A-D). The top 1200 genes that were most associated with ARL14 were selected for enrichment analysis using the clusterProfiler $\mathrm{R}$ package. In LUAD, GO analysis revealed that ARL14 was primarily involved in cell growth and differentiation terms, including epidermal development, cell-substrate junction, cell adhesion mediator activity, cell adhesion molecule binding, and cell junction organization (Figure 7A-C). Moreover, the related genes were involved in focal adhesion and NOD-like receptor signaling pathways, according to KEGG functional enrichment analysis (Figure 7D). We also conducted GO terms and KEGG pathway enrichment in LUSC, which indicated that ARL14 was mainly related to DNA replication (Figure 7E-H). Figure 7A-H shows the most significant GO terms for biological process, cellular component, and molecular function, as well as KEGG pathways.

GSEA was performed to identify the signaling pathways that are activated in LUAD and LUSC. The results showed that pathways for neutrophil degranulation, signaling by interleukins, and extracellular matrix organization were differentially enriched in the positively correlated with ARL14 expression phenotype in LUSC. For LUAD, gene sets related to keratinization, positive epigenetic regulation of rRNA expression, and deacetylate histones (HDACS) showed differential enrichment in the high ARL14 gene expression phenotype (Figure 8).

\section{Correlation Between Immune Cell Infiltration and ARLI4}

Tumor-infiltrating lymphocytes affect the survival of patients with various cancers. Thus, we further analyzed the correlation between the expression of ARL14 and immune infiltration by ssGSEA with Spearman $r$. The correlation between ARL14 expression levels and 24 immune cells was observed in the forest plots (Figure 9A and E). The results showed that ARL14 expression was negatively correlated with infiltration levels of follicular helper $\mathrm{T}$ cells $(\mathrm{TFH})(\mathrm{P}<0.001$; Figure $9 \mathrm{~B})$, and positively correlated with that of activated dendritic cells (aDC) and T-helper 1 cells $($ Th1) $(\mathrm{P}<0.001$; Figure 9C and D) in LUAD. However, there was no significant correlation between ARL14 expression and the number of infiltrated TFH cells $(\mathrm{P}=0.4$; Figure $9 \mathrm{~F})$ in LUSC. The results showed that the expression level of ARL14 was positively correlated with infiltrating levels of neutrophils ( $\mathrm{P}<$ $0.001)$ and Th1 cells $(\mathrm{P}<0.001)$ in LUSC (Figure 9G and $\mathrm{H}$ ).

\section{Discussion}

Lung cancer is one of the most common diseases among humans, ranking at the top for the diagnosis and deaths in both sexes combined according to the database generated by the International Agency of Research on Cancer (IARC) Global Cancer Observatory in 2018. ${ }^{1}$ Patients with NSCLC account for the majority of lung cancer cases. It is essential to identify biomarkers for tumorigenesis and poor outcomes in NSCLC.

ARL14, a member of the Ras superfamily, is reported to have an important relationship with the occurrence and development of human tumors. ${ }^{13}$ The ARL14 protein is involved in apoptosis signaling and several regulatory pathways. ${ }^{25-30}$ Its homologous similar genes play an important role in human tumors; for example, ARF4 is involved in breast cancer cell migration as reported by Jang et al. ${ }^{31}$

Our study indicated that ARL14 protein appeared to be a promising candidate as a therapeutic target and prognostic predictor. Using bioinformatics analysis based on online datasets, survival analysis revealed the prognostic value of ARL14 in NSCLC. Indeed, this result was consistent with our IHC analysis and survival analysis results of the included patients with NSCLC.

Inflammation marked by the recruitment of leukocytes and HDACS reversibly regulates the acetylation status of histone and non-histone proteins, which are considered to be correlated with the development and metastasis of cancer. $^{32,33}$ GSEA showed that inflammation-related pathways such as neutrophil degranulation and signaling 

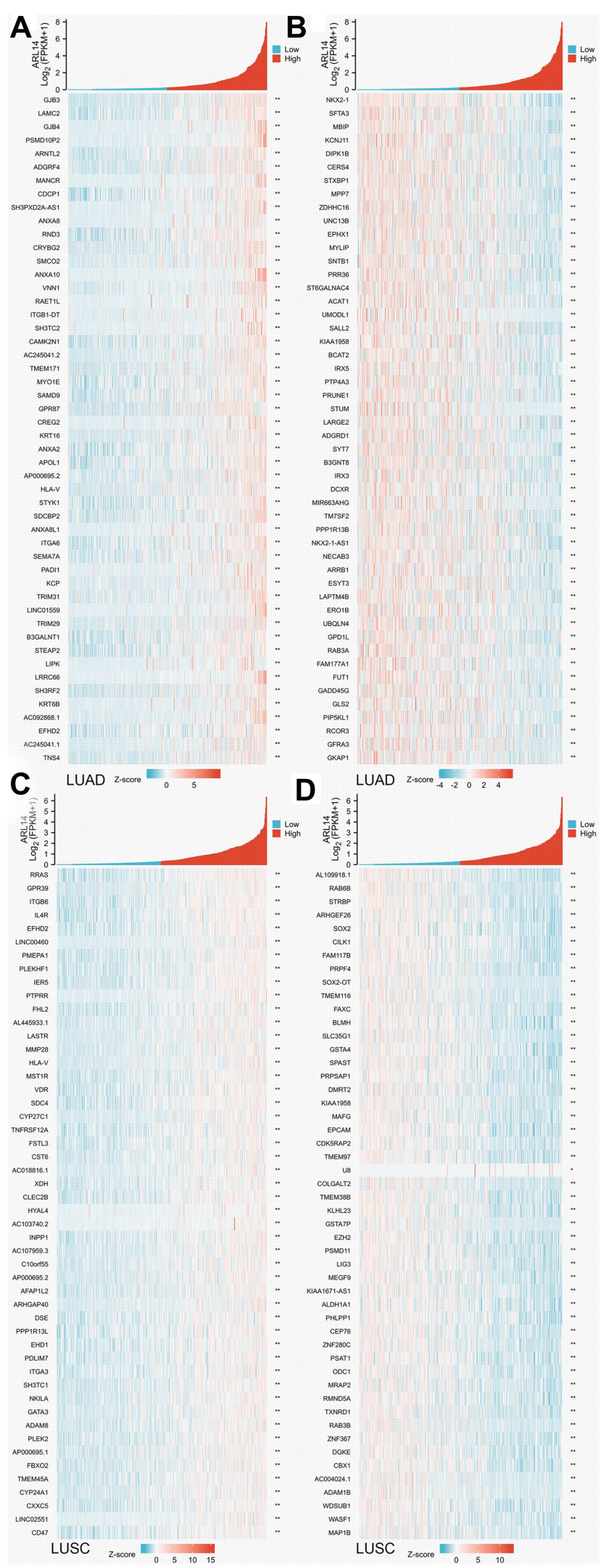

Figure 6 Correlation analysis of ARLI4 in LUAD and LUSC. (A) Top 50 genes most positively associated with ARLI4 in patients with LUAD were shown in a heatmap. (B) Top 50 genes most negatively associated with ARLI 4 in patients with LUAD were shown in a heatmap. (C) Top 50 genes most positively associated with ARLI 4 in patients with LUSC were shown in a heatmap. (D) Top 50 genes most negatively associated with ARLI 4 in patients with LUSC were shown in a heatmap. ${ }^{p}<0.05$; ** $p<0.00$ I. 

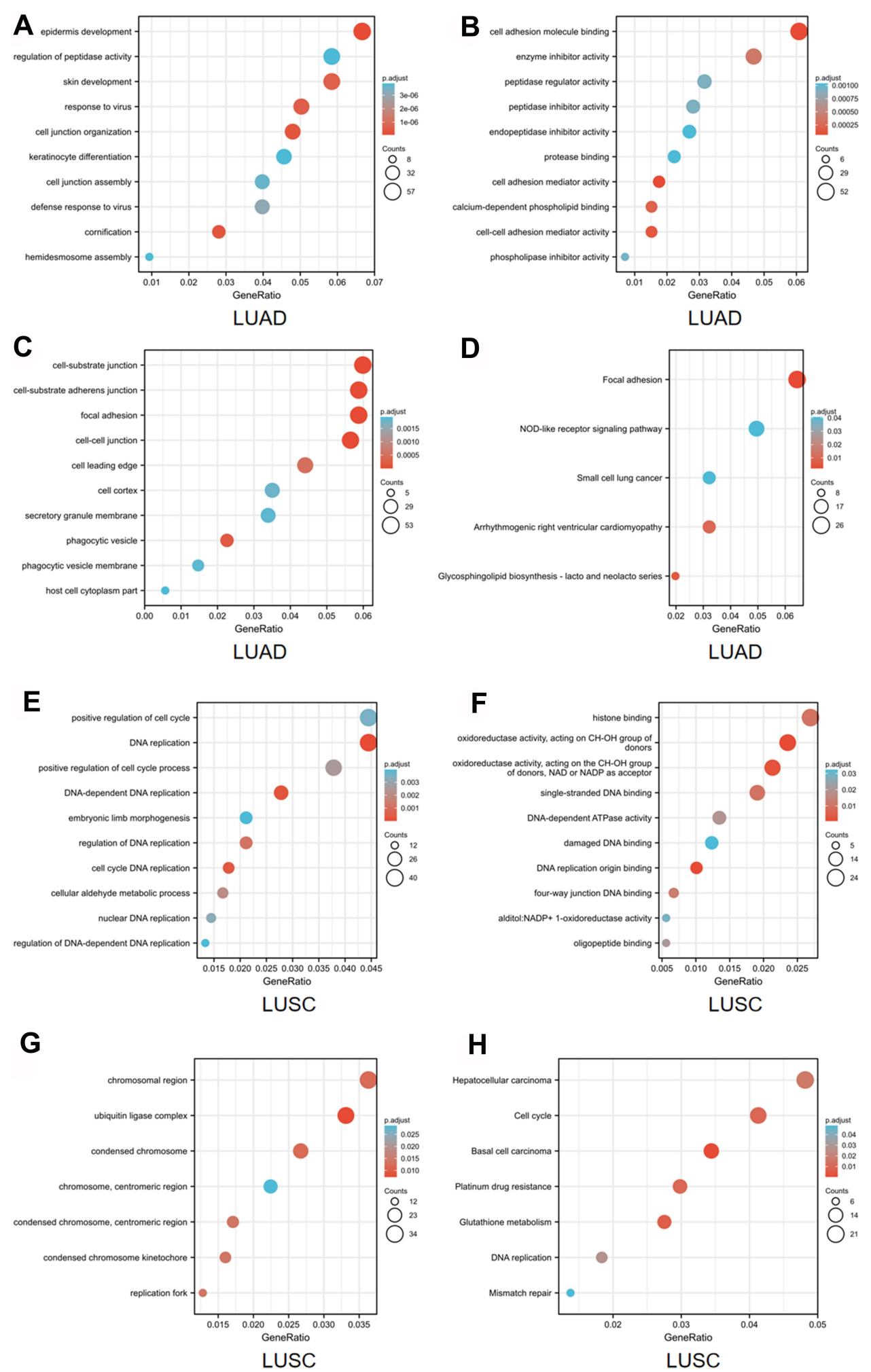

Figure 7 Enrichment analysis of ARLI 4 in LUAD and LUSC. (A-C) Significant Gene Ontology terms of the top I 200 genes most associated with ARLI 4 in LUAD, including BP, MF, and CC. (D) Significant KEGG pathways of the top 1200 genes most associated with ARLI4 in LUAD. (E-G) Significant Gene Ontology terms of the top I200 genes most associated with ARLI4 in LUSC, including BP, MF, and CC. (H) Significant KEGG pathways of the top 1200 genes most associated with ARLI4 in LUSC.

by interleukins were positively correlated with ARL14 expression phenotype in LUSC, and HDACS showed differential enrichment in the high ARL14 gene expression phenotype in LUAD. Thus, ARL14 expression may be related to the occurrence and development of tumors. 


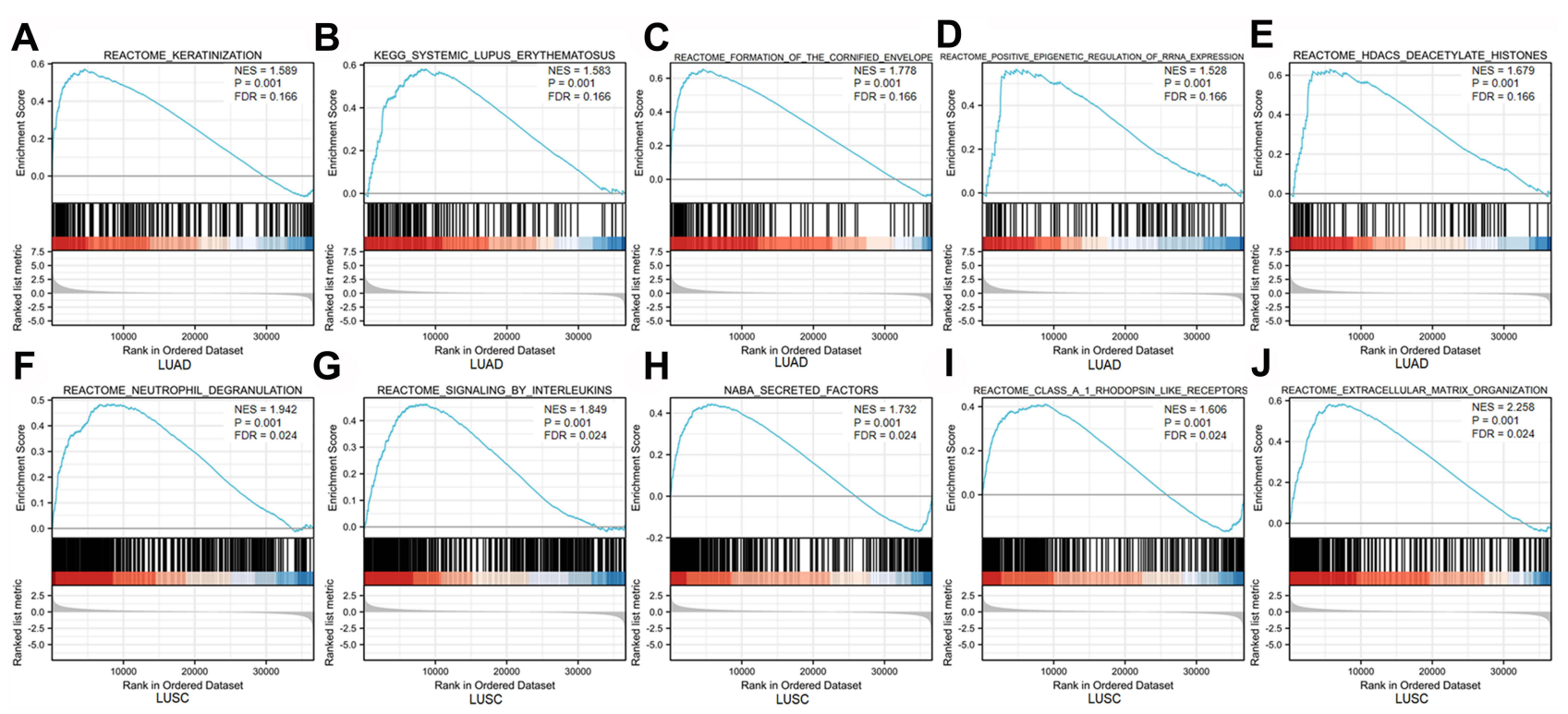

Figure 8 Enrichment plot from the GSEA. Enrichment plots of (A) keratinization, (B) systemic lupus erythematosus, (C) formation of the cornified envelope, (D) positive epigenetic regulation of RRNA expression, and (E) HDACS deacetylate histones in LUAD cases with high ARLI4 expression. Gene set enrichment plots of (F) neutrophil degranulation, (G) signaling by interleukins, (H) NABA secreted factors, (I) class AI rhodopsin like receptors, and (J) extracellular matrix organization in LUAD or LUSC cases with high ARLI4 expression.

Abbreviations: NES, normalized NS; FDR, false discovery rate.
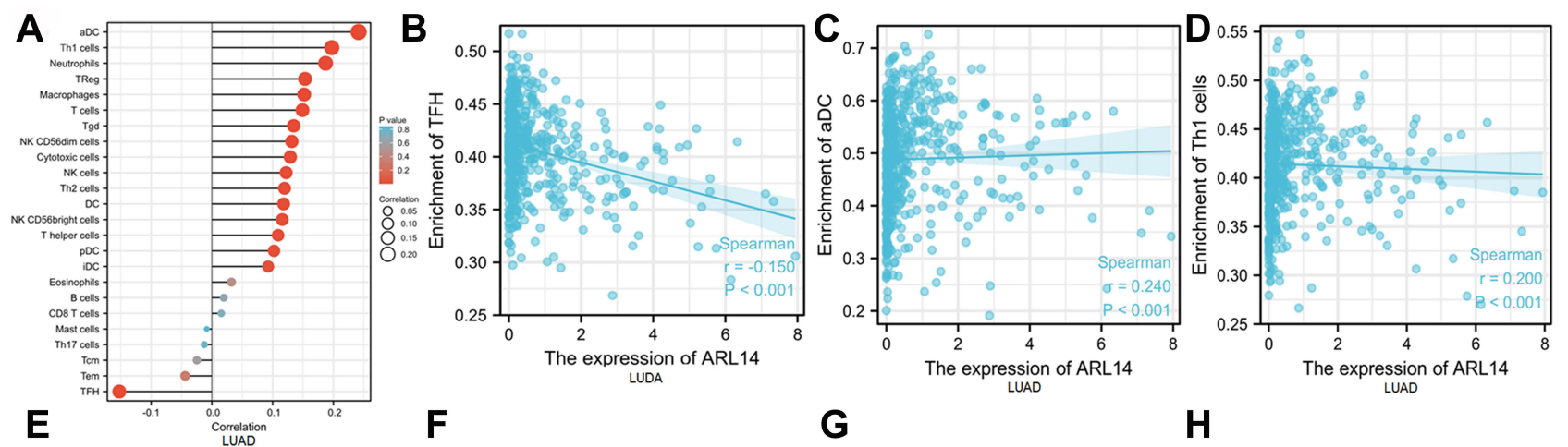

\section{$\mathbf{F}$}

G

H
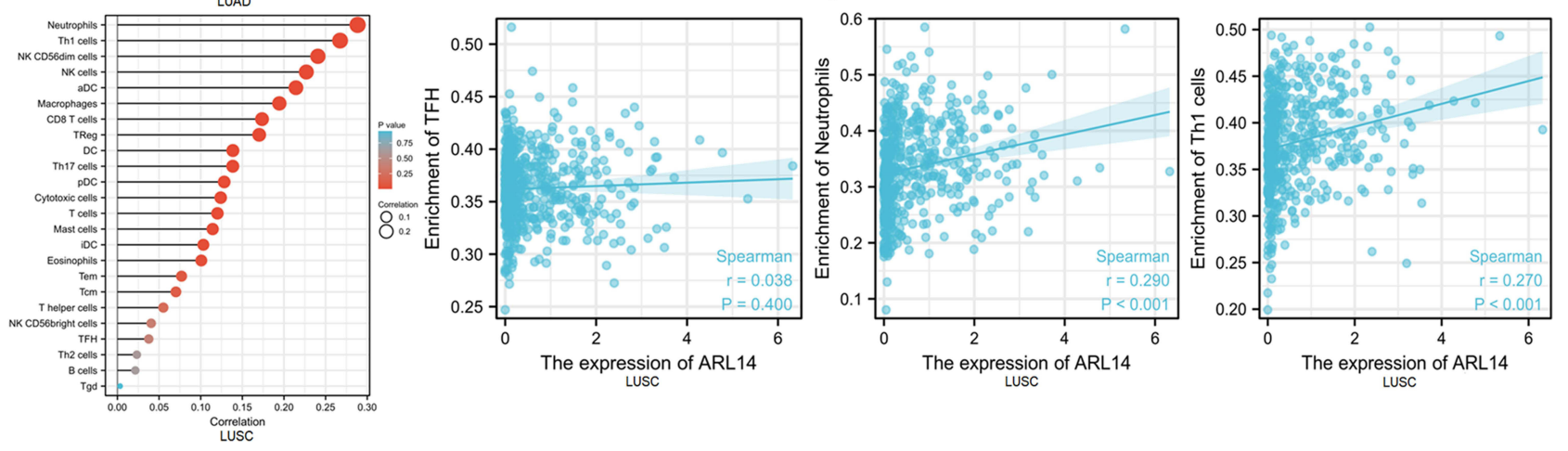

Figure 9 Correlation between immune cell infiltration and ARLI4 in LUAD and LUSC. The forest plots showed the association between ARLI4 expression and 24 immune cells in LUAD (A) and LUSC (E), respectively. (B) The relationship between ARLI 4 expression and TFH in LUAD. (C) The relationship between ARLI4 expression and aDC in LUAD. (D) The relationship between ARLI4 expression and ThI in LUAD. (F) The relationship between ARLI4 expression and TFH in LUSC. (G) The relationship between ARLI4 expression and neutrophils in LUSC. (H) The relationship between ARLI4 expression and ThI in LUSC. 
Tumors use several immune processes to escape immune surveillance such as targeting regulatory $\mathrm{T}$ cell function or its secretions and changing the production of immunosuppressive mediators. ${ }^{34}$ The invasion of NSCLC is related to immune regulation functions of the body such as changes in the Th1/Th2 cytokine ratio and T-cell infiltration. ${ }^{34-36}$ Our study indicated that the level of ARL14 was positively correlated with infiltrating levels of Th1 cells and T cells in LUAD and LUSC, which was consistent with previous reports. The high number and abnormal function of TFH cells can be related to immunosuppression and development of tumors in NSCLC..$^{37,38}$ In addition, the TFH1 subtype is negatively correlated with PFS after tumor resection in NSCLC. ${ }^{38}$ Our study indicated that ARL14 expression was negatively correlated with TFH cell infiltration in LUAD and had no significant correlation in LUSC. Thus, further studies related to immunity in NSCLC need to be conducted.

Although we did not further study the specific mechanism of ARL14 in the occurrence and development of NSCLC, there have been reports that high ARL14 expression promotes the development of LUAD through the CIDEC/ERK/p38 signaling pathway. Guo et al demonstrated that silencing ARL14 could block ERK1/2 and p38 signaling and stimulate its downstream gene CIDEC expression, finally resulting in cell cycle arrest in LUAD cells. ${ }^{39}$ Their in vitro experiments revealed that ARL14 could serve as a prognostic marker and a valuable therapeutic target for LUAD patients. Regrettably, there is still a lack of information on the mechanism of ARL14 in NSCLC. Therefore, further studies on ARL14 in lung cancer are needed.

\section{Conclusion}

In summary, by employing bioinformatics analysis based on online datasets, our study indicated high ARL14 levels in NSCLC. By collecting clinical samples, we focused on immunohistochemical analysis and survival analysis of ARL14 protein in NSCLC. The results indicated a significant correlation between ARL14 expression and NSCLC prognosis.

\section{Ethics Approval and Consent to Participate}

This study was approved by Medical Ethics Committee of the First Affiliated Hospital of Anhui Medical University according to the guidelines of the Declaration of Helsinki. Informed consent was obtained from each participant.

\section{Acknowledgments}

The authors thank Professor Wenning Wu and Professor Daxiong Zeng for providing guidance.

\section{Funding}

This research was supported by the fund for Natural Science Foundation of China (No.81970051), Excellent Top Talent Cultivation Project of Anhui Higher Education Institutions (gxgwfx2021014), Scientific Research Fund from Anhui Medical University (2020xkj257) and Applied Medical Research Project of Hefei Health Commission (Hwk2021zd008).

\section{Disclosure}

The authors have no conflicts of interest in this work.

\section{References}

1. Bray F, Ferlay J, Soerjomataram I, Siegel RL, Torre LA, Jemal A. Global cancer statistics 2018: GLOBOCAN estimates of incidence and mortality worldwide for 36 cancers in 185 countries. CA Cancer J Clin. 2018;68(6):394-424. doi:10.3322/caac.21492

2. Miller KD, Nogueira L, Mariotto AB, et al. Cancer treatment and survivorship statistics, 2019. CA Cancer J Clin. 2019;69(5):363-385. doi:10.3322/caac. 21565

3. Relli V, Trerotola M, Guerra E, Alberti S. Abandoning the notion of non-small cell lung cancer. Trends Mol Med. 2019;25(7):585-594. doi:10.1016/j.molmed.2019.04.012

4. Ettinger DS, Wood DE, Aggarwal C, et al. NCCN guidelines insights: non-small cell lung cancer, version 1.2020. J Natl Compr Canc Netw. 2019;17(12):1464-1472. doi:10.6004/jncen.2019.0059

5. Liu Y, Zhou S, Du Y, et al. Efficacy and safety of programmed death 1 inhibitors in patients with advanced non-small cell lung cancer: a meta-analysis. Cancer Manag Res. 2019;11:4619-4630. doi:10.2147/ CMAR.S193394

6. Zhang B, Liu Y, Zhou S, Jiang H, Zhu K, Wang R. Predictive effect of PD-L1 expression for immune checkpoint inhibitor (PD-1/PD-L1 inhibitors) treatment for non-small cell lung cancer: a meta-analysis. Int Immunopharmacol. 2020;80:106214. doi:10.1016/j.intimp.2020. 106214

7. Liu Y, Li HM, Wang R. Effectiveness and safety of adding bevacizumab to platinum-based chemotherapy as first-line treatment for advanced non-small-cell lung cancer: a meta-analysis. Front Med. 2021;8:616380. doi:10.3389/fmed.2021.616380

8. Paul P, van den Hoorn T, Jongsma ML, et al. A genome-wide multidimensional RNAi screen reveals pathways controlling MHC class II antigen presentation. Cell. 2011;145(2):268-283. doi:10.1016/j.cell. 2011.03.023

9. Ota T, Suzuki Y, Nishikawa T, et al. Complete sequencing and characterization of 21,243 full-length human cDNAs. Nat Genet. 2004;36(1):40-45. doi:10.1038/ng1285

10. Donaldson JG, Jackson CL. ARF family G proteins and their regulators: roles in membrane transport, development and disease. Nat Rev Mol Cell Biol. 2011;12(6):362-375. doi:10.1038/nrm3117

11. Tanna CE, Goss LB, Ludwig CG, Chen PW. Arf GAPs as regulators of the actin cytoskeleton-an update. Int $J$ Mol Sci. 2019;20:2. doi:10.3390/ijms20020442

12. Gillingham AK, Munro S. The small G proteins of the Arf family and their regulators. Annu Rev Cell Dev Biol. 2007;23:579-611. doi:10.1146/annurev.cellbio.23.090506.123209 
13. Colicelli J. Human RAS superfamily proteins and related GTPases. Sci STKE. 2004;2004(250):Re13. doi:10.1126/stke.2502004re13

14. Gaudet P, Livstone MS, Lewis SE, Thomas PD. Phylogenetic-based propagation of functional annotations within the Gene Ontology consortium. Brief Bioinform. 2011;12(5):449-462. doi:10.1093/bib/ bbr042

15. Wang L, Shi J, Huang Y, et al. A six-gene prognostic model predicts overall survival in bladder cancer patients. Cancer Cell Int. 2019;19:229. doi:10.1186/s12935-019-0950-7

16. Liu J, Liu S, Wu M, et al. ARF3 inhibits proliferation and promotes apoptosis in gastric cancer by regulating AKT and ERK pathway. Acta biochimica Polonica. 2021;68(2):223-229.

17. Liang C, Qin Y, Zhang B, et al. ARF6, induced by mutant Kras, promotes proliferation and Warburg effect in pancreatic cancer. Cancer Lett. 2017;388:303-311. doi:10.1016/j.canlet.2016.12.014

18. Rami-Porta R, Bolejack V, Crowley J, et al. The IASLC lung cancer staging project: proposals for the Revisions of the $\mathrm{T}$ descriptors in the forthcoming eighth edition of the TNM classification for lung cancer. $J$ Thorac Oncol. 2015;10(7):990-1003. doi:10.1097/JTO.000000000 0000559

19. Wang R, Zhou S, Wu P, et al. Identifying involvement of H19-miR675-3p-IGF1R and H19-miR-200a-PDCD4 in treating pulmonary hypertension with melatonin. Mol Ther Nucleic Acids. 2018;13:44-54. doi:10.1016/j.omtn.2018.08.015

20. Zhou S, Jiang H, Li M, et al. Circular RNA hsa_circ_0016070 is associated with pulmonary arterial hypertension by promoting PASMC proliferation. Mol Ther Nucleic Acids. 2019;18:275-284. doi:10.1016/j.omtn.2019.08.026

21. Bai Y, Liu X, Qi X, et al. PDIA6 modulates apoptosis and autophagy of non-small cell lung cancer cells via the MAP4K1/JNK signaling pathway. EBioMedicine. 2019;42:311-325. doi:10.1016/j.ebiom.2019.03.045

22. Yu G, Wang LG, Han Y, He QY. clusterProfiler: an R package for comparing biological themes among gene clusters. OMICS. 2012;16 (5):284-287. doi:10.1089/omi.2011.0118

23. Hänzelmann S, Castelo R, Guinney J. GSVA: gene set variation analysis for microarray and RNA-seq data. BMC Bioinform. 2013;14:7. doi:10.1186/1471-2105-14-7

24. Ito K, Murphy D. Application of ggplot2 to pharmacometric graphics. CPT: Pharmacomet Syst Pharmacol. 2013;2(10):e79.

25. Yang F, Li T, Peng Z, Liu Y, Guo Y. The amphipathic helices of Arfrp1 and Arl14 are sufficient to determine subcellular localizations. $J$ Biol Chem. 2020;295(49):16643-16654. doi:10.1074/jbc.RA120.014999

26. Ninomiya H, Kato M, Sanada M, et al. Allelotypes of lung adenocarcinomas featuring ALK fusion demonstrate fewer onco- and suppressor gene changes. BMC Cancer. 2013;13:8. doi:10.1186/14712407-13-8
27. Du M, Thompson J, Fisher H, Zhang P, Huang CC, Wang L. Genomic alterations of plasma cell-free DNAs in small cell lung cancer and their clinical relevance. Lung Cancer. 2018;120:113-121. doi:10.1016/j.lungcan.2018.04.008

28. Litviakov NV, Cherdyntseva NV, Tsyganov MM, et al. Deletions of multidrug resistance gene loci in breast cancer leads to the down-regulation of its expression and predict tumor response to neoadjuvant chemotherapy. Oncotarget. 2016;7(7):7829-7841. doi:10.18632/oncotarget.6953

29. Barrios-Rodiles M, Brown KR, Ozdamar B, et al. High-throughput mapping of a dynamic signaling network in mammalian cells. Science. 2005;307(5715):1621-1625. doi:10.1126/science.1105776

30. Kahn RA, Der CJ, Bokoch GM. The ras superfamily of GTP-binding proteins: guidelines on nomenclature. FASEB J. 1992;6 (8):2512-2513. doi:10.1096/fasebj.6.8.1592203

31. Jang SY, Jang SW, Ko J. Regulation of ADP-ribosylation factor 4 expression by small leucine zipper protein and involvement in breast cancer cell migration. Cancer Lett. 2012;314(2):185-197. doi:10.1016/j.canlet.2011.09.028

32. Mollinedo F. Neutrophil degranulation, plasticity, and cancer metastasis. Trends Immunol. 2019;40(3):228-242. doi:10.1016/j. it.2019.01.006

33. Li Y, Seto E. HDACs and HDAC inhibitors in cancer development and therapy. Cold Spring Harb Perspect Med. 2016;6(10):a026831. doi:10.1101/cshperspect.a026831

34. Vinay DS, Ryan EP, Pawelec G, et al. Immune evasion in cancer: mechanistic basis and therapeutic strategies. Semin Cancer Biol. 2015;35:S185-s198. doi:10.1016/j.semcancer.2015.03.004

35. Anichini A, Perotti VE, Sgambelluri F, Mortarini R. Immune escape mechanisms in non small cell lung cancer. Cancers. 2020;12 (12):3605. doi:10.3390/cancers12123605

36. Stankovic B, Bjørhovde HAK, Skarshaug R, et al. Immune cell composition in human non-small cell lung cancer. Front Immunol. 2018;9:3101. doi:10.3389/fimmu.2018.03101

37. Ng KW, Marshall EA, Enfield KS, et al. Somatic mutation-associated $\mathrm{T}$ follicular helper cell elevation in lung adenocarcinoma. Oncoimmunology. 2018;7(12):e1504728. doi:10.1080/2162402X. 2018.1504728

38. Qiu L, Yu Q, Zhou Y, et al. Functionally impaired follicular helper $\mathrm{T}$ cells induce regulatory B cells and CD14(+) human leukocyte antigen-DR(-) cell differentiation in non-small cell lung cancer. Cancer Sci. 2018;109(12):3751-3761. doi:10.1111/cas.13836

39. Guo F, Yuan D, Zhang J, et al. Silencing of ARL14 gene induces lung adenocarcinoma cells to a dormant state. Front Cell Dev Biol. 2019;7:238. doi:10.3389/fcell.2019.00238
Journal of Inflammation Research

\section{Publish your work in this journal}

The Journal of Inflammation Research is an international, peerreviewed open-access journal that welcomes laboratory and clinical findings on the molecular basis, cell biology and pharmacology of inflammation including original research, reviews, symposium reports, hypothesis formation and commentaries on: acute/chronic inflammation; mediators of inflammation; cellular processes; molecular mechanisms; pharmacology and novel anti-inflammatory drugs; clinical conditions involving inflammation. The manuscript management system is completely online and includes a very quick and fair peerreview system. Visit http://www.dovepress.com/testimonials.php to read real quotes from published authors. 